Construction of multiple trade-offs to obtain arbitrary singularities of adaptive dynamics

Kisdi, Eva

2015-04

Kisdi , E 2015 , ' Construction of multiple trade-offs to obtain arbitrary singularities of adaptive dynamics ' , Journal of mathematical biology , vol. 70 , no. 5 , pp. 1093-1117 . https://doi.org/10.1007/s0028

http://hdl.handle.net/10138/262548

https://doi.org/10.1007/s00285-014-0788-5

acceptedVersion

Downloaded from Helda, University of Helsinki institutional repository.

This is an electronic reprint of the original article.

This reprint may differ from the original in pagination and typographic detail.

Please cite the original version. 


\title{
Construction of multiple trade-offs to obtain arbitrary singularities of adaptive dynamics
}

\author{
Eva Kisdi ${ }^{1}$
}

This article has been published

This is the Author Version of an article published in the Journal of Mathematical Biology. The final publication is available at Springer via http://dx.doi.org/10.1007/s00285-0140788-5.

\section{Please cite as:}

Kisdi E. 2015. Construction of multiple trade-offs to obtain arbitrary singularities of adaptive dynamics. J. Math. Biol. 70: 1093-1117.

Keywords: critical function analysis, evolutionary branching, evolutionarily singular strategy, coexistence, trade-off, host-pathogen-predator system

Mathematics Subject Classification: 92D15, 92D30, 92D40

\begin{abstract}
Evolutionary singularities are central to the adaptive dynamics of evolving traits. The evolutionary singularities are strongly affected by the shape of any trade-off functions a model assumes, yet the trade-off functions are often chosen in an ad hoc manner, which may unjustifiably constrain the evolutionary dynamics exhibited by the model. To avoid this problem, critical function analysis has been used to find a trade-off function that yields a certain evolutionary singularity such as an evolutionary branching point. Here I extend this method to multiple trade-offs parameterized with a scalar strategy. I show that the trade-off functions can be chosen such that an arbitrary point in the viability domain of the trait space is a singularity of an arbitrary type, provided (next to certain non-degeneracy conditions) that the model has at least two environmental feedback variables and at least as many trade-offs as feedback variables. The proof is constructive, i.e., it provides an algorithm to find trade-off functions that yield the desired singularity. I illustrate the construction of trade-offs with an example where the virulence of a pathogen evolves in a small ecosystem of a host, its pathogen, a predator that attacks the host and an alternative prey of the predator.
\end{abstract}

\footnotetext{
${ }^{1}$ Department of Mathematics and Statistics, University of Helsinki, PO Box 68, FIN-00014 Finland; Tel.: +358-50-448 0277, email: eva.kisdi@helsinki.fi
} 


\section{Introduction}

Trade-off functions linking various traits subject to selection are ubiquitous in models of evolutionary ecology. The precise shapes of these trade-off functions are, however, often the least substantiated assumptions of the models. Analytical tractability may require that simple formulas represent the trade-offs, but this still leaves considerable freedom for choice, and the choices made are usually not backed up by empirical evidence. Indeed, in many cases the empirical data are too noisy to establish the shape of a trade-off function in sufficient detail; yet the details matter for the predictions of the models (see e.g. de Mazancourt and Dieckmann 2004 and Geritz et al. 2007 for examples).

Critical function analysis is an increasingly popular technique used in adaptive dynamics to circumvent the necessity of assuming trade-off functions in an ad hoc manner (de Mazancourt and Dieckmann 2004; Kisdi 2006; see Bowers et al. 2005 for a closely related approach, and Geritz et al. 2007; Svennungsen and Kisdi 2009; Boldin et al. 2009; Zu et al. 2011; Morozov and Best 2012 for some applications). Instead of starting with a certain trade-off function and deriving the location and properties of evolutionary singularities based on this particular tradeoff, critical function analysis finds the slope (first derivative) of the trade-off at a given point such that this point is singular, and establishes whether the convexity (second derivative) of the trade-off can be chosen such that the singularity is of a desired type, for example such that evolutionary branching occurs. This analysis is carried out at each point of the trait space that corresponds to a viable population. The procedure thus either yields concrete examples for the trade-off function that lead to a certain singularity (such as a branching point), or else proves that this type of singularity cannot occur in the model with any choice of the trade-off function.

The existing technique of critical function analysis considers only a single trade-off, and as a consequence, it has only limited control over the properties of the evolutionary singularities it delivers (see the Discussion for details). In this paper, I extend critical function analysis to multiple trade-offs between traits determined by an underlying scalar strategy. First I consider a general model of adaptive dynamics and give sufficient conditions under which a monomorphic singularity of any desired type can be obtained at any given point in (the viability domain of) the trait space by choosing appropriate trade-off functions. Note that the conditions found in the first part of the paper are sufficient but not necessary, i.e., failing these conditions does not imply that some type of singularity may not occur in the model. The proof is constructive, i.e., when the sufficient conditions are satisfied, it gives a method to construct trade-off functions that make an arbitrary point of the trait space a singularity of an arbitrary type.

In the second part of the paper I describe a worked example, where I investigate evolutionary branching of pathogens when their host is subject to predation. Next to illustrating the general results, I also show how a direct method of analysis can be applied to concrete models; the general results of the first part ensure that the direct method of constructing trade-offs delivers the desired singularity. The direct method can also be used to confirm or disprove that a certain singularity occurs under biologically plausible trade-offs (see Discussion). Readers who wish to study the construction method but wish to avoid some technicalities can read the direct analysis of the worked example in sections 4.1 and 4.3 without the rest of the main text. 


\section{Preliminaries}

Let $r_{x}(y)$ denote the invasion fitness of the mutant strategy $y$ in the resident population of strategy $x\left(x, y \in X \subseteq \mathbb{R}\right.$; Metz et al. 1992). $x$ is singular if $\left.\frac{\partial r}{\partial y}\right|_{y=x}=0$. Coexistence by mutual invasibility occurs in the neighbourhood of the singularity if $\mathcal{M}=\left.\frac{\partial^{2} r_{x}(y)}{\partial x \partial y}\right|_{y=x}<0$, whereas if $\mathcal{M}>0$, then there are pairs of strategies in the neighbourhood of the singularity that mutually exclude each other (Geritz et al. 1998; note that as long as $\mathcal{M} \neq 0$, coexistence near a singularity is possible only by mutual invasibility, Geritz 2005). The singularity is evolutionarily stable (sensu Maynard Smith 1982) if $\mathcal{E}=\left.\frac{\partial^{2} r_{x}(y)}{\partial y^{2}}\right|_{y=x}<0$ and it is convergence stable if $\mathcal{E}+\mathcal{M}<0$ (Eshel 1983). The full classification of generic monomorphic evolutionary singularities of scalar strategies, given by Geritz et al. (1998), is rewritten in Table 1 in terms of $\mathcal{M}$ and $\mathcal{E}$.

Table 1. Generic types of monomorphic evolutionary singularities of scalar strategies (based on Geritz et al. 1998).

\begin{tabular}{|c|c|c|c|c|c|}
\hline Singularity $^{1}$ & $\begin{array}{l}\text { Conv. } \\
\text { stab. }^{3}\end{array}$ & $\begin{array}{l}\text { Evol. } \\
\text { stab. }\end{array}$ & $\begin{array}{l}\text { Inv. } \\
\text { cap. }\end{array}$ & $\begin{array}{l}\text { Mut. } \\
\text { inv. }\end{array}$ & Condition \\
\hline (b) Branching point & yes & no & yes & yes & $\mathcal{M}<-\mathcal{E}<0$ \\
\hline (c) $\mathrm{CSS}^{2}$ & yes & yes & yes & yes & $\mathcal{M}<0$ and $\mathcal{E}<0$ \\
\hline (d) CSS & yes & yes & yes & no & $0<\mathcal{M}<-\frac{\mathcal{E}}{2}$ \\
\hline (e) CSS & yes & yes & no & no & $0<-\frac{\mathcal{E}}{2}<\mathcal{M}<-\mathcal{E}$ \\
\hline (f) Garden of Eden & no & yes & no & no & $0<-\mathcal{E}<\mathcal{M}$ \\
\hline (g) Non-ESS repellor ${ }^{2}$ & no & no & no & no & $\mathcal{M}>0$ and $\mathcal{E}>0$ \\
\hline (h) Non-ESS repellor & no & no & no & yes & $-\frac{\mathcal{E}}{2}<\mathcal{M}<0$ \\
\hline (a) Non-ESS repellor & no & no & yes & yes & $-\mathcal{E}<\mathcal{M}<-\frac{\mathcal{E}}{2}<0$ \\
\hline
\end{tabular}

${ }^{1}$ The letters (a)-(h) correspond to the pairwise invasibility plots shown in Figure 2 of Geritz et al. (1998), who classified the singularities in terms of the second derivatives $\left.\frac{\partial^{2} r}{\partial x^{2}}\right|_{y=x}$ and $\mathcal{E}=\left.\frac{\partial^{2} r}{\partial y^{2}}\right|_{y=x} ;$ to switch to $\mathcal{M}$ and $\mathcal{E}$, note that $r_{x}(x)=0$ for all $x \in X$ and this implies $\left.\frac{\partial^{2} r}{\partial x^{2}}\right|_{y=x}+2 \mathcal{M}+\mathcal{E}=0$.

${ }^{2}$ The three types of CSSs and three types of non-ESS repellors are distinguished by their invading capability and whether mutual invasibility exists in their neighbourhoods.

3 "Conv. stab.", convergence stability; "Evol. stab.", evolutionary stability; "Inv. cap.", invading capability (whether the singular strategy can invade when itself is rare in the resident population of a strategy in its neighbourhood); "Mut. inv.", mutual invasibility near the singularity ("no" means mutual exclusion). Note that the four properties are pairwise independent but not fully independent.

Suppose that the scalar strategy determines $m$ observable trait values, $f_{1}, \ldots, f_{m}$. The parametric curve $x \mapsto\left(f_{1}(x), \ldots, f_{m}(x)\right)$ corresponds, at least locally (away from critical points), to $m-1$ trade-off functions connecting the observable traits (figure 1). I assume that the trait 
vector $\mathbf{f}(x)=\left[f_{1}(x), \ldots, f_{m}(x)\right]^{T}$ is a twice continuously differentiable function of the strategy $x$ ( $T$ denotes the transpose). The trade-off structure is characterized locally by the derivative vectors $\mathbf{f}^{\prime}(x)=d \mathbf{f}(x) / d x$ and $\mathbf{f}^{\prime \prime}(x)=d^{2} \mathbf{f}(x) / d x^{2}$ (throughout, I shall refer to $x \mapsto \mathbf{f}(x)$ as the trade-off structure, in order to distinguish it from the individual trade-offs as the one in figure 1). Instead of assuming a certain trade-off structure a priori and differentiating it to obtain $\mathbf{f}^{\prime}(x)$ and $\mathbf{f}^{\prime \prime}(x)$, I seek vectors $\mathbf{f}^{\prime}(x)$ and $\mathbf{f}^{\prime \prime}(x)$ such that the trade-off structure yields the desired singularity. More precisely, given a strategy $x$ with an arbitrary trait vector $\mathbf{f}(x)$, the goal is to find vectors $\mathbf{f}^{\prime}(x)$ and $\mathbf{f}^{\prime \prime}(x)$ such that if

$$
\mathbf{f}(y)=\mathbf{f}(x)+\mathbf{f}^{\prime}(x)(y-x)+\mathbf{f}^{\prime \prime}(x)(y-x)^{2}+O\left((y-x)^{3}\right)
$$

then $x$ is a singularity of a given type (for example, $x$ is an evolutionary branching point). I consider trade-off structures only with $\mathbf{f}^{\prime}(x) \neq \mathbf{0}$, i.e., I require that at least some trait(s) change with $x$ in first order. Because we are free to scale $x$, the vector $\mathbf{f}^{\prime}(x)$ is defined only up to a multiplicative constant.

The invasion fitness of a rare mutant strategy $y$ in the resident population of strategy $x$ is rewritten in terms of the observable traits as

$$
r_{x}(y)=s\left(f_{1}(y), \ldots, f_{m}(y), E_{1}, \ldots, E_{n}\right)
$$

where $E_{i}=E_{i}\left(f_{1}(x), \ldots, f_{m}(x)\right)$ denotes the $i$ th environmental feedback variable, such as the abundance of a resource, the density of a predator, etc., as determined by the resident population of strategy $x$ at equilibrium. I assume that the feedbacks $E_{i}\left(f_{1}, \ldots, f_{m}\right)$ are continuously differentiable and the invasion fitness $s\left(f_{1}, \ldots, f_{m}, E_{1}, \ldots, E_{n}\right)$ is twice continuously differentiable in all their arguments. $E_{1}, \ldots, E_{n}$ contain all ecological variables that are affected by the resident population, and that in turn affect the invasion fitness of a mutant, such that the strategies influence each other's population growth exclusively through the $n$ environmental feedback variables (Mylius and Diekmann 1995; Diekmann et al. 2001, 2003; Meszéna et al. 2006; Metz et al. 2008). I assume that the population dynamics of the resident attains a hyperbolic fixed point attractor (multiple attractors of population dynamics can be accommodated using the results of Geritz et al. 2002).

Let $s_{i}$ denote the partial derivative of $s\left(f_{1}, \ldots, f_{m}, E_{1}, \ldots, E_{n}\right)$ with respect to its $i$ th argument evaluated at the resident traits $(\mathbf{f}=\mathbf{f}(x))$, and let the column vectors $\mathbf{z}=\left[s_{1}, \ldots, s_{m}\right]^{T}$

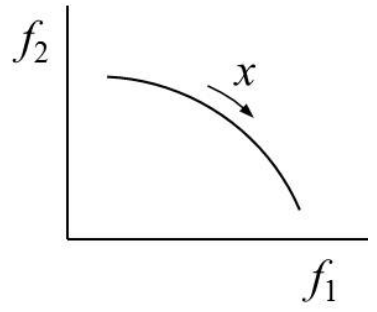

Figure 1: The scalar strategy $x$ generates a trade-off between two observable traits, $f_{1}(x)$ and $f_{2}(x)$. 
and $\mathbf{w}=\left[s_{m+1}, \ldots, s_{m+n}\right]^{T}$ collect respectively the partial derivatives of the invasion fitness with respect to traits and with respect to feedbacks. Because the resident population size is regulated through the environmental feedbacks, $\mathbf{w} \neq \mathbf{0}$ must hold at the hyperbolic point attractor of the resident population dynamics.

Further, let $E_{i, j}$ be the partial derivative of the $i$ th feedback, $E_{i}\left(f_{1}, \ldots, f_{m}\right)$, with respect to the $j$ th trait evaluated at the resident's trait values, and let the $n \times m$ matrix $\mathbf{E}$ collect the numbers $E_{i, j}$. Because

$$
s\left(f_{1}, \ldots, f_{m}, E_{1}\left(f_{1}, \ldots, f_{m}\right), \ldots, E_{n}\left(f_{1}, \ldots, f_{m}\right)\right)=0
$$

for all viable trait vectors $\mathbf{f}$, from implicit differentiation with respect to $f_{i}$, we have $s_{i}+$ $\sum_{j=1}^{n} s_{m+j} E_{j, i}=0$ for $i=1, \ldots, m$, i.e.,

$$
\mathbf{z}^{T}+\mathbf{w}^{T} \mathbf{E}=\mathbf{0}^{T}
$$

Lastly, let $\mathbf{S}$ be the $m \times n$ matrix of $S_{i, j}=s_{i, m+j}=\frac{\partial s_{i}}{\partial E_{j}}=\frac{\partial^{2} s}{\partial f_{i} \partial E_{j}}$ evaluated at the resident. The vectors and matrices $\mathbf{z}, \mathbf{w}, \mathbf{E}$ and $\mathbf{S}$ depend on the resident trait vector $\mathbf{f}(x)$, but this is suppressed in the notation; similarly, $\mathbf{f}(x), \mathbf{f}^{\prime}(x)$ and $\mathbf{f}^{\prime \prime}(x)$ will often be abbreviated as $\mathbf{f}, \mathbf{f}^{\prime}$ and $\mathbf{f}^{\prime \prime}$, respectively, and these shorthand notations will always mean vectors consisting of numbers, not functions.

I make the following assumptions about the traits and environmental feedback variables to hold at least locally in the neighbourhood of the trait vector $\mathbf{f}(x)$ of the resident strategy $x$ :

(A1) The traits may depend arbitrarily on the strategy in the sense that $\mathbf{f}^{\prime}(x)$ and $\mathbf{f}^{\prime \prime}(x)$ may be any vector in $\mathbb{R}^{m}$.

(A2) Each trait impacts at least some environmental feedback variables in first order, i.e., $\mathbf{E}$ has no zero column.

(A1) excludes that traits are listed repeatedly or some traits are fixed combinations of others; including such traits would inflate the number of traits without any change to the biological system. Importantly, (A1) implies that we are free to postulate any trade-off structure. Traits that violate (A2) have no impact on the invasion fitness, because by (4), $E_{1, i}=\ldots=E_{n, i}=0$ implies $s_{i}=0$ as well. (A2) can thus be replaced with the slightly stronger assumption

(A2') Each trait impacts the invasion fitness in first order, i.e., $s_{i} \neq 0$ for $i=1, \ldots, m$,

which is easier to check. If (A2) or (A2') is violated only at an isolated point, then the corresponding trait vector $\mathbf{f}(x)$ is a critical point of the model, but one can easily avoid this degeneracy by choosing a different trait vector to work with. If however (A2) is violated in an open set of trait vectors and environments, then the violating trait is a selectively neutral trait, which inflates the number of traits without affecting fitness. Many selectively neutral traits exist in reality, but these are not counted among the $m$ traits in the invasion fitness (2) even if they depend on strategy $x$. (A2) also implies that the resident strategy is viable. If the resident 
population goes extinct, then the environmental feedback variables return to the state of the "virgin environment", where they are independent of the traits of the (absent) resident.

Finally, I rewrite the singularity condition and the second derivatives $\mathcal{M}$ and $\mathcal{E}$ in terms of the observable traits, using the invasion fitness function in (2). The strategy $x$ is singular if $\left.\frac{\partial r}{\partial y}\right|_{y=x}=\sum_{i=1}^{m} s_{i} f_{i}^{\prime}(x)=0$, i.e., if $\mathbf{z}^{T} \mathbf{f}^{\prime}=0$; with equation (4), this can be rewritten as

$$
\mathbf{w}^{T} \mathbf{E} \mathbf{f}^{\prime}=0 .
$$

$\mathcal{M}$ evaluates to

$$
\mathcal{M}=\left.\frac{\partial^{2} r}{\partial x \partial y}\right|_{y=x}=\sum_{i=1}^{m} f_{i}^{\prime} \sum_{j=1}^{n} \sum_{k=1}^{m} \frac{\partial s_{i}}{\partial E_{j}} E_{j, k} f_{k}^{\prime}=\mathbf{f}^{\prime T} \mathbf{S E f ^ { \prime }}
$$

whereas $\mathcal{E}$ is given by

$$
\mathcal{E}=\left.\frac{\partial^{2} r}{\partial y^{2}}\right|_{y=x}=\sum_{i=1}^{m} \sum_{j=1}^{m} s_{i, j} f_{i}^{\prime} f_{j}^{\prime}+\sum_{i=1}^{m} s_{i} f_{i}^{\prime \prime}
$$

(note that $s_{i, j}$ is different from $S_{i, j}=s_{i, m+j}$ ).

\section{Results}

In this section, I explore conditions under which trade-offs can be found locally in the neighbourhood of a given strategy $x$, i.e., the vectors $\mathbf{f}^{\prime}(x)$ and $\mathbf{f}^{\prime \prime}(x)$ in (1) can be chosen to a given trait vector $\mathbf{f}(x)$, such that $x$ is an evolutionarily singular strategy of a desired type. In the first two propositions, I give sufficient conditions under which $\mathbf{f}^{\prime}(x)$ can be chosen such that $x$ is singular with $\mathcal{M}=0$, and sufficient conditions under which this degenerate singularity can be unfolded into a singularity at the same strategy $x$ but with $\mathcal{M}<0$ (an analogous procedure yields $\mathcal{M}>0$ ). The third proposition is based on existing results and shows that once $x$ is singular with the appropriate sign of $\mathcal{M}, \mathbf{f}^{\prime \prime}(x)$ can be chosen such that also $\mathcal{E}$ satisfies the inequality conditions of the desired singularity (see Table 1).

First I seek a vector $\mathbf{f}^{\prime} \neq \mathbf{0}$ that gives $\mathcal{M}=0$ in (6) and also satisfies the singularity condition (5). Without specific model assumptions, the matrices $\mathbf{E}$ and $\mathbf{S}$ are unknown. For example if SE (or its symmetric part) happens to be positive definite or negative definite, then there is no nonzero vector $\mathbf{f}^{\prime}$ that satisfies $\mathcal{M}=0$; and even if $\mathbf{S E}$ has a nontrivial nullspace, it might be outside of the $m-1$ dimensional subspace defined by the singularity condition (5). The first proposition below ensures the existence of the required trade-off structure, provided that there are at least as many trade-offs as environmental feedbacks $(m-1 \geq n)$. The idea of the proof is that any vector $\mathbf{f}^{\prime}$ that satisfies $\mathbf{E} \mathbf{f}^{\prime}=\mathbf{0}$ immediately satisfies the singularity condition (5) and gives $\mathcal{M}=0$ in (6). With $m>n$, the system $\mathbf{E f ^ { \prime }}=\mathbf{0}$ is underdetermined such that a nonzero solution can be found.

Proposition 1. At any given $x$ and $\mathbf{f}(x)$ where (A1)-(A2) are satisfied, $\mathbf{f}^{\prime}(x) \neq \mathbf{0}$ can be chosen such that $x$ is singular with $\mathcal{M}=0$ with the trade-off structure in (1), provided that $m>n$. 
Proof. Let $k$ denote the rank of $\mathbf{E}(k \leq n)$. Assume, without loss of generality, that the first $k$ columns of $\mathbf{E}$ are linearly independent and partition $\mathbf{E}$ as $\mathbf{E}=\left[\mathbf{E}_{\mathbf{1}} \mid \mathbf{E}_{\mathbf{2}}\right]$ with an $n \times k$ block $\mathbf{E}_{\mathbf{1}}$ and an $n \times(m-k)$ block $\mathbf{E}_{\mathbf{2}}$. Correspondingly, let $\mathbf{f}^{\prime T}=\left[\mathbf{f}_{\mathbf{1}}^{\prime T} \mid \mathbf{f}_{\mathbf{2}}^{\prime T}\right]$ where $\mathbf{f}_{\mathbf{1}}^{\prime}$ is a $k$-vector and $\mathbf{f}_{\mathbf{2}}^{\prime}$ is an $(m-k)$-vector. $m>n$ implies that $\mathbf{E}_{\mathbf{2}}$ has at least one column and $\mathbf{f}_{\mathbf{2}}^{\prime}$ has at least one element. To find a vector $\mathbf{f}^{\prime}$ that satisfies the singularity condition (5) with $\mathcal{M}=0$, we seek a vector that satisfies $\mathbf{E f}^{\prime}=\mathbf{0}$, or, equivalently,

$$
\mathbf{E}_{1} \mathbf{f}_{1}^{\prime}=-\mathbf{E}_{2} \mathbf{f}_{2}^{\prime}
$$

$\mathbf{f}_{\mathbf{2}}^{\prime} \neq \mathbf{0}$ can be chosen arbitrarily. If the right hand side $\mathbf{r}=-\mathbf{E}_{\mathbf{2}} \mathbf{f}_{\mathbf{2}}^{\prime}$ is the zero vector, then $\mathbf{f}_{\mathbf{1}}^{\prime}=\mathbf{0}$ is the trivial solution of (8). If $\mathbf{r}$ is not the zero vector, then the inhomogeneous system $\mathbf{E}_{\mathbf{1}} \mathbf{f}_{\mathbf{1}}^{\prime}=\mathbf{r}$ contains $n$ equations for the $k \leq n$ elements of $\mathbf{f}_{\mathbf{1}}^{\prime}$, yet it always has a solution: By the assumption that all $k$ linearly independent columns of $\mathbf{E}$ are in the block $\mathbf{E}_{\mathbf{1}}$, one can write the columns of $\mathbf{E}_{\mathbf{2}}$ as linear combinations of the columns of $\mathbf{E}_{\mathbf{1}}$, i.e., $\mathbf{E}_{\mathbf{2}}=\mathbf{E}_{\mathbf{1}} \mathbf{A}$ with some $k \times(m-k)$ matrix $\mathbf{A}$. The solution to (8) is then given by $\mathbf{f}_{\mathbf{1}}^{\prime}=-\mathbf{A} \mathbf{f}_{\mathbf{2}}^{\prime}$, and any trade-off structure in (1) with $\mathbf{f}^{\prime T}(x)=\left[-\mathbf{A} \mathbf{f}_{\mathbf{2}}^{\prime T} \mid \mathbf{f}_{\mathbf{2}}^{\prime T}\right]$ makes $x$ singular with $\mathcal{M}=0$.

Remark 1. Assumption (A2) is technically not necessary for Proposition 1 to hold. However, if only one element of $\mathbf{f}^{\prime}$ is nonzero, then the resulting trade-off curve is degenerate as all $d f_{i} / d f_{j}=f_{i}^{\prime} / f_{j}^{\prime}$ are zero or infinite or undefined (in the latter case, $d f_{i} / d f_{j}$ depends on higher derivatives of $f_{i}$ and $f_{j}$ ). If (A2) holds, then $\mathbf{f}_{\mathbf{2}}^{\prime} \neq \mathbf{0}$ can be chosen such that $\mathbf{r}=-\mathbf{E}_{\mathbf{2}} \mathbf{f}_{\mathbf{2}}^{\prime}$ is not the zero vector; with $\mathbf{E}_{\mathbf{2}}=\mathbf{E}_{\mathbf{1}} \mathbf{A}$, this implies that $\mathbf{f}_{\mathbf{2}}^{\prime}$ is not in the nullspace of $\mathbf{A}$, i.e., $\mathbf{f}_{\mathbf{1}}^{\prime}=-\mathbf{A} \mathbf{f}_{\mathbf{2}}^{\prime}$ is not the zero vector. Because both $\mathbf{f}_{\mathbf{1}}^{\prime}$ and $\mathbf{f}_{\mathbf{2}}^{\prime}$ contain at least one nonzero element, at least two elements of $\mathbf{f}^{\prime}$ are different from zero, such that the corresponding traits exhibit a trade-off with nonzero and finite slope (whereas traits corresponding to any zero elements of $\mathbf{f}^{\prime}$ may be assumed constant). While (A2) is sufficient to achieve this, it is not necessary; if for example $\mathbf{E}_{\mathbf{2}}$ contains two zero columns, then the two corresponding elements of $\mathbf{f}_{\mathbf{2}}^{\prime}$ can be chosen nonzero. A trade-off between two selectively neutral traits is however biologically uninteresting, and the resulting singularity with $\mathcal{M}=0$ cannot be unfolded (see Remark 11).

Remark 2. $\mathbf{E}$ is not full rank $(k<n)$ if some environmental feedbacks can be expressed with others. As seen from the proof above, increasing rank deficiency increases the number of elements in $\mathbf{f}^{\prime}$ that can be chosen arbitrarily. This increasing freedom is however not necessary for finding a suitable trade-off structure. The sufficient condition $m>n$ is the sharpest if $\left(E_{1}, \ldots, E_{n}\right)$ is a minimal representation of the environmental feedback, such that $\mathbf{E}$ is full rank.

Remark 3. The singularity is isolated if $\mathcal{E}+\mathcal{M} \neq 0$. As Proposition 3 shows below, $\mathbf{f}^{\prime \prime}(x)$ can be chosen such that $\mathcal{E} \neq 0$, which makes the singularity obtained in Proposition 1 isolated.

Remark 4. Notice that $\mathbf{E f ^ { \prime }}=\mathbf{0}$ implies that each feedback variable has a critical point as a function of the strategy $x$, i.e., $d E_{i} / d x=\sum_{j} E_{i, j} f_{j}^{\prime}=0$ for $i=1, \ldots, n$.

Next I show that the singularity with $\mathcal{M}=0$, constructed as in Proposition 1, can generically be unfolded by changing $\mathbf{f}^{\prime}(x)$ into $\mathbf{f}^{\prime}(x)+\Delta \mathbf{f}^{\prime}(x)$ such that $x$ remains singular but with $\mathcal{M}<0$, provided that $m \geq n$ and there are at least two environmental feedback variables. This unfolding yields a trade-off structure under which there are strategy pairs near $x$ that can coexist by mutual invasibility. $\mathcal{M}>0$ and mutual exclusion can be obtained analogously. 
Proposition 2. Let $\mathbf{E} \mathbf{f}^{\prime}(x)=\mathbf{0}$ such that $x$ is singular with $\mathcal{M}=0$. Suppose $m \geq n \geq 2$. Then a vector $\Delta \mathbf{f}^{\prime}(x)$ can be found such that when $\mathbf{f}^{\prime}(x)$ is replaced with $\mathbf{f}^{\prime}(x)+\Delta \mathbf{f}^{\prime}(x)$ in (1), $x$ is singular with $\mathcal{M}<0$, provided that two non-degeneracy conditions are satisfied:

(i) $\operatorname{rank}(\mathbf{E}) \geq 2$ and

(ii) $\mathbf{S}^{T} \mathbf{f}^{\prime}(x) \neq k \mathbf{w}$ (where $k \in \mathbb{R}$ is arbitrary).

Proof. Let $\mathbf{u}$ denote the $n$-vector $\mathbf{E} \Delta \mathbf{f}^{\prime}$. Differentiating (6) with respect to $\mathbf{f}^{\prime}$ and using $\mathbf{E} \mathbf{f}^{\prime}=\mathbf{0}$ yields $\frac{d \mathcal{M}}{d \mathbf{f}^{\prime}}=\mathbf{f}^{\prime T} \mathbf{S E}$, i.e., an infinitesimal change $\Delta \mathbf{f}^{\prime}$ changes $\mathcal{M}$ by

$$
\Delta \mathcal{M}=\mathbf{f}^{\prime T} \mathbf{S E} \Delta \mathbf{f}^{\prime}=\mathbf{f}^{\prime T} \mathbf{S u}
$$

It suffices to show that $\Delta \mathbf{f}^{\prime}$ can be chosen such that $\Delta \mathcal{M}$ is nonzero while the singularity condition $\mathbf{w}^{T} \mathbf{E}\left(\mathbf{f}^{\prime}+\Delta \mathbf{f}^{\prime}\right)=\mathbf{w}^{T} \mathbf{E} \Delta \mathbf{f}^{\prime}=\mathbf{w}^{T} \mathbf{u}=0$ holds (cf (5)); if $\Delta \mathcal{M}$ is positive, replace $\Delta \mathbf{f}^{\prime}$ with $-\Delta \mathbf{f}^{\prime}$. First one needs to find a vector $\mathbf{u}$ that is orthogonal to $\mathbf{w}$ and is not orthogonal to $\mathbf{S}^{T} \mathbf{f}^{\prime}$; this is always possible if $\mathbf{S}^{T} \mathbf{f}^{\prime} \neq k \mathbf{w}$. The unfolding vector $\Delta \mathbf{f}^{\prime}$ is the solution of the $n$ equations $\mathbf{E} \Delta \mathbf{f}^{\prime}=\mathbf{u}$ for the $m$ elements of $\Delta \mathbf{f}^{\prime}$. If $\mathbf{E}$ is full rank, then with $m \geq n$ a solution exists. If $n=2$, then $\mathbf{E}$ is full rank by condition (i). If $n>2$ and $\mathbf{E}$ is not full rank, then the system $\mathbf{E} \Delta \mathbf{f}^{\prime}=\mathbf{u}$ may contain contradicting equations for some choices of $\mathbf{u}$. However, $(n-2)$ elements of $\mathbf{u}$ can be chosen arbitrarily (because $\mathbf{u}$ is constrained only by $\mathbf{w}^{T} \mathbf{u}=0$ and $\mathbf{f}^{\prime T} \mathbf{S u} \neq 0$ ). Since $\operatorname{rank}(\mathbf{E}) \geq 2, \mathbf{u}$ can be chosen such that the equations do not contradict (in other words, since at least 2 rows of $\mathbf{E}$ are linearly independent, there are no more than $n-2$ potentially contradicting equations, such that all contradictions can be avoided by choosing $n-2$ elements of $\mathbf{u}$ appropriately). Thus with $m \geq n$, a solution for $\Delta \mathbf{f}^{\prime}$ exists.

Remark 5. Condition (i) is a non-degeneracy condition (i.e., it excludes only exceptional cases) under the main assumption of Proposition 2 that $m \geq n \geq 2$, i.e., $\mathbf{E}$ is at least a $2 \times 2$ matrix. (ii) is a non-degeneracy condition when the main assumption $n \geq 2$ holds, so that $\mathbf{S}^{T} \mathbf{f}^{\prime}(x)$ and w are not scalars.

Remark 6. Note that Proposition 2 assumes $m \geq n$, whereas Proposition 1 assumes the strict inequality $m>n$. If $m=n$, then one cannot guarantee by Proposition (1) that a singularity with $\mathbf{E} \mathbf{f}^{\prime}=\mathbf{0}$ (and hence $\mathcal{M}=0$ ) exists, but if it does, then one can unfold it when the other conditions of Proposition 2 are satisfied. For the entire construction method to work, one has to assume $m>n$ such that Proposition 1 can be used to obtain the degenerate singularity to be unfolded using Proposition 2.

Remark 7. When the construction method is applied to a concrete model, we may wish to identify the strategy with one of the observable traits, i.e., we may wish to assume $f_{i}(x)=x$ for some $i$. To maintain this identity through the construction, we need $f_{i}^{\prime}=1$ and $\Delta f_{i}^{\prime}=0$. Since $\mathbf{f}^{\prime}$ constructed according to Proposition 1 is determined only up to a multiplicative constant, it is easy to scale $\mathbf{f}^{\prime}$ such that $f_{i}^{\prime}=1$ holds, provided that $f_{i}^{\prime}$ is nonzero (which is true for at least some $i$ ). If $m>n$ as required by Proposition 1, then there is an extra degree of freedom for choosing $\Delta \mathbf{f}^{\prime}$ in Proposition 2, which can be used to set $\Delta f_{i}^{\prime}=0$ (see the next section for an example). 
The next four remarks discuss the role of environmental feedback variables. As it is well known, both mutual invasibility and mutual exclusion are impossible if the model has only a single environmental feedback variable (Metz et al. 2008). In this case $\mathcal{M}=0$, and none of the generic evolutionary singularities can occur (cf. Table 1).

Remark 8. If there is a single environmental feedback variable $(n=1)$, then $\mathbf{w}$ and $\mathbf{S}^{T} \mathbf{f}^{\prime}$ are scalars, and because $\mathbf{S}^{T} \mathbf{f}^{\prime}=k \mathbf{w}$ holds for some $k$ (recall that $\mathbf{w} \neq \mathbf{0}$ ), $\mathcal{M}=0$ cannot be unfolded. With $n=1$, the singularity condition implies $\mathbf{E} \Delta \mathbf{f}^{\prime}=0$, and therefore $\Delta \mathcal{M}=0$.

Remark 9. Suppose that there are $n>1$ environmental feedback variables but the invasion fitness depends only on a single scalar-valued function of these, i.e., the invasion fitness can be written in the form $s\left(f_{1}(y), \ldots, f_{m}(y), h\left(E_{1}, \ldots, E_{n}\right)\right)$ with some function $h: \mathbb{R}^{n} \rightarrow \mathbb{R}$. In this case, $S_{i, j}=\frac{\partial^{2} s}{\partial f_{i} \partial E_{j}}=h_{j} \frac{\partial^{2} s}{\partial f_{i} \partial h}$ where $h_{j}=\frac{\partial h}{\partial E_{j}}$ is a constant, which implies that $S_{i, l}=\left(\frac{h_{l}}{h_{j}}\right) S_{i, j}$ for all $i, j, l$ and therefore $\operatorname{rank}(\mathbf{S})=1$. It is easy to see that the 1-dimensional range of $\mathbf{S}^{T}$ contains $\mathbf{w}=\left(\frac{\partial s}{\partial h}\right)\left[h_{1}, \ldots, h_{n}\right]^{T}$ such that $\mathbf{S}^{T} \mathbf{f}^{\prime}=k \mathbf{w}$ for some $k$, and $\mathcal{M}=0$ cannot be unfolded. The reason is that the effective number of environmental feedbacks is only 1 (see Metz et al. 2008).

Remark 10. The matrix $\mathbf{E}$ is the derivative of the function $\left(f_{1}, \ldots, f_{m}\right) \mapsto\left(E_{1}, \ldots, E_{n}\right)$ at the point $\mathbf{f}(x)$, and therefore the rank of $\mathbf{E}$ shows the dimension of the image of the function when its arguments are varied in an infinitesimal open neighbourhood of $\mathbf{f}(x)$. If $\operatorname{rank}(\mathbf{E})=k<n$, then the environments $\left(E_{1}, \ldots, E_{n}\right)$ that are generated by some resident trait vectors are in a $k$-dimensional hyperplane, and the remaining $n-k$ transformed variables are constants that can be subsumed into the fitness function $s$. Since the feedback environment can (locally) be given in terms of only $k$ variables (Metz et al. 2008), the effective number of environmental feedbacks is at most the rank of $\mathbf{E}$ (it may be less, see Remark 9 ). If $\operatorname{rank}(\mathbf{E})=1$, then $\mathcal{M}=0$ cannot be unfolded because the effective number of environmental feedbacks is only 1.

Remark 11. Suppose that $\operatorname{rank}(\mathbf{E})=k$ and $m-k$ traits are selectively neutral such that $m-k$ columns of $\mathbf{E}$ are zero and (A2') is violated for $i=k+1, \ldots, m$ in an open neighbourhood of the point $\left(f_{1}(x), \ldots f_{m}(x), E_{1}, \ldots E_{n}\right)$ (with $E_{i}=E_{i}\left(f_{1}(x), \ldots, f_{m}(x)\right)$ ). The latter implies $S_{i, j}=0$ for all $j$ when $i=k+1, \ldots, m$, i.e., the last $m-k$ columns of $\mathbf{S}^{T}$ are zero. By Proposition $1, \mathbf{f}^{\prime} \neq \mathbf{0}$ can be found, but its first $k$ elements are zero. It follows that $\mathbf{S}^{T} \mathbf{f}^{\prime}=\mathbf{0}=k \mathbf{w}$ with $k=0$, which implies that $\mathcal{M}=0$ cannot be unfolded.

The last remark highlights a connection with short-term population dynamics (this is not essential for the rest of the paper).

Remark 12. Two theorems by Priklopil (2012) connect the unfolding of $\mathcal{M}=0$ with the shortterm population dynamics of two coexisting resident strategies as follows (see Priklopil (2012) for details). (i) For $\epsilon>0$ sufficiently small, the invasion boundaries of the pairwise invasibility plot generically intersect in the neighbourhood of the singularity either when $\mathcal{M}=\epsilon$ or when $\mathcal{M}=-\epsilon$. (ii) The intersection of invasion boundaries implies the existence of multiple population dynamic attractors (unprotected coexistence) for some pairs of strategies near the intersection. Since the unfolding of $\mathcal{M}=0$ described in this paper yields singularities both with $\mathcal{M}=\epsilon$ and with $\mathcal{M}=-\epsilon$, it produces trade-offs under which populations with two resident strategies have multiple population dynamic attractors.

Of the singularity condition, $\mathcal{M}$, and $\mathcal{E}$, only $\mathcal{E}$ depends on the vector of second derivatives $\mathbf{f}^{\prime \prime}(x)$ (cf. (5), (6) and (7)). This means that one can tune the value of $\mathcal{E}$ by changing only 
$\mathbf{f}^{\prime \prime}(x)$ such that the singularity condition and $\mathcal{M}$ are not affected. Since $\mathcal{E}$ is linear in $\mathbf{f}^{\prime \prime}$, we immediately have

Proposition 3. Suppose that $\mathbf{f}^{\prime}(x)$ is given. The vector of second derivatives $\mathbf{f}^{\prime \prime}(x)$ can be chosen to obtain an arbitrary value of $\mathcal{E}$ when $\mathbf{z} \neq \mathbf{0}$. In particular, once $\mathbf{f}^{\prime}(x)$ is such that $x$ is singular and the sign of $\mathcal{M}$ is as required by the desired type of singularity, $\mathbf{f}^{\prime \prime}(x)$ can be chosen such that $\mathcal{E}$ satisfies the remaining inequality conditions of the singularity (cf Table 1).

Proposition 3 corresponds to the existing technique of critical function analysis. For a single trade-off, this result was shown by Kisdi (2006), based on the work of de Mazancourt and Dieckmann (2004) and Bowers et al. (2005). With multiple trade-offs, one may fix all but one element of $\mathbf{f}^{\prime \prime}(x)$ and choose the remaining $f_{i}^{\prime \prime}(x)$ to achieve the same, provided that $s_{i} \neq 0$. Under assumption (A2'), any one element can be used.

In summary, one can find trade-offs that make $x$ an evolutionarily singular strategy of an arbitrarily chosen type under mild non-degeneracy conditions, provided that there are at least two environmental feedback variables and more traits than feedbacks. It is well known that at least two environmental feedbacks are necessary for either mutual invasibility or mutual exclusion near the singularity, and hence for all generic types of singularities in Table 1, when the resident system attains a population dynamic equilibrium (Levin 1970; McGehee and Armstrong 1977; Meszéna et al. 2006; Metz et al. 2008). The new result is that given this (and the non-degeneracy conditions), there are always trade-off structures that make an arbitrary point in trait space a singularity of an arbitrary type, if the number of traits involved in the trade-offs exceeds the number of environmental feedbacks. Here the traits may obviously not include selectively neutral traits, repeated traits or combinations of other traits (see assumptions (A1) and (A2)). The number of environmental feedbacks needs two important qualifications: the effective number of feedbacks is less than the number of listed variables if some variables act only through a fixed function of them (Remark 9), and also if the environments generated by the possible resident trait vectors do not span the whole space (Remark 10). Derivatives of third and higher order do not influence the properties of generic evolutionary singularities, therefore every trade-off structure with the same $\left(\mathbf{f}(x), \mathbf{f}^{\prime}(x), \mathbf{f}^{\prime \prime}(x)\right)$ has the same type of singularity at $x$. The local trade-off structure given up to second order in (1) can therefore be extended over an arbitrary interval of resident strategies in any biologically plausible way.

\section{Example: Evolutionary branching of pathogens under preda- tion of their hosts}

In this section, I illustrate the above general results with a model for the evolution of pathogen virulence, where I seek evolutionary branching of the pathogen. This example is a generalized version of the models studied by Morozov and Best (2012) and Kisdi et al. (2013). 


\subsection{The model}

I assume that the host of the pathogen is embedded in a small ecosystem of three species: the host, a predator that may preferentially attack hosts weakened by the infection, and an alternative prey of the predator. The pathogen can infect only the host species, and the host has no acquired immunity (SIS model). The ecological dynamics are given by the equations

$$
\begin{aligned}
\frac{d S}{d t} & =b(N) N-(\mu+c P) S-\beta S I+\nu I \\
\frac{d I}{d t} & =[\beta S-(k \alpha+\mu+\nu)-(c+\phi) P] I \\
\frac{d Z}{d t} & =[\rho(1-Z / K)-\zeta P] Z \\
\frac{d P}{d t} & =[\gamma c S+\gamma(c+\phi) I+\theta \zeta Z-\delta] P
\end{aligned}
$$

where $S, I, Z$ and $P$ are respectively the population densities of susceptible hosts, infected hosts, the alternative prey and the predator, and $N=S+I$ is total host density. Hosts are born at a density-dependent per capita birth rate $b$ and all newborns are susceptible. Susceptible hosts have a background mortality rate $\mu$, are captured by a predator at a rate $c$ per predator, and are infected at a rate $\beta$ per infected (mass action). Infected hosts recover at a rate $\nu$ and die due to the disease at a rate $k \alpha$, where $\alpha$ is the virulence of the disease. I introduce the factor $k$ in order to be able to change the importance of direct mortality relative to the elevated predation risk of the infected ( $k=0$ corresponds to a disease that does not kill the host but may make it more vulnerable to predation). $\phi$ is the extra predation rate (per predator) towards infected hosts. The alternative prey of the predator follows logistic population growth with intrinsic growth rate $\rho$ and carrying capacity $K$, and the predator attacks the alternative prey at a rate $\zeta$. The predator converts the consumed hosts and alternative prey into predator offspring with conversion factors $\gamma$ and $\theta$, respectively (for simplicity, I assume that healthy and infected hosts are equally nutritious for the predator). The predator dies at a constant rate $\delta$.

The population dynamical equilibrium and its stability depend on the yet unspecified per capita birth rate function $b$. Unfortunately, even the simplest choices of $b$ yield very large formulas for the equilibrium value of $N$. For this reason, in the analytical part I leave $b$ unspecified and therefore the equilibrium host density implicit, assuming throughout that the equilibrium densities are positive and the equilibrium is stable. Appendix A shows that the equilibrium is always asymptotically stable if $b^{\prime}(N)$ is sufficiently large at the equilibrium value of $N$. In the numerical example presented below, I assume a logistic host with linear density dependence in its per capita birth rate $\left(b(N)=b_{0}-q N\right)$, and check the positivity of the equilibrium and the eigenvalues of the Jacobian numerically.

The pathogen is characterized by three traits $(m=3)$, the virulence $(\alpha)$, the transmission rate $(\beta)$ and how much an infected host is weakened, measured by the extra capture rate of predators towards infected hosts $(\phi)$. For simplicity, I assume the recovery rate to be fixed. Assuming complete cross-immunity, a mutant strain of the pathogen spreads according to

$$
\frac{d I_{m u t}}{d t}=\left[\beta_{m u t} S-\left(k \alpha_{m u t}+\mu+\nu\right)-\left(c+\phi_{m u t}\right) P\right] I_{m u t} .
$$


The three observable traits of the pathogen depend on an underlying scalar strategy. The model can be formulated such that virulence itself is the strategy $(x=\alpha)$ and the other two traits depend on virulence (see Remark 7); with a slight abuse of notation, I write $\beta_{\text {mut }}=\beta\left(\alpha_{m u t}\right)$ and $\phi_{m u t}=\phi\left(\alpha_{m u t}\right)$, where $\beta$ and $\phi$ are the two trade-off functions. The invasion fitness of the mutant is the expression in the brackets in (11). The invasion fitness depends on two environmental feedback variables set by the resident system $(n=2)$, the density of susceptibles $(S)$ and the density of predators $(P)$.

The next section will apply the general results obtained in Section 3 to this example. However, when working with a concrete model, one can obtain the same (and slightly more) results from direct calculations, bypassing the matrix formulation of Section 3. This direct analysis is presented in section 4.3. Readers who wish to study the construction method through a worked example rather than the general results can thus skip section 4.2 and proceed to 4.3 .

\subsection{Application of the general results}

The basic ingredients of the general model are the three-dimensional trait vector $\mathbf{f}=[\alpha, \beta, \phi]^{T}$ and the two environmental feedback variables $E_{1}=S$ and $E_{2}=P$. Applying the definitions of $\mathbf{w}$ and $\mathbf{S}$ to the invasion fitness

$$
s(\alpha, \beta, \phi, S, P)=\beta S-(k \alpha+\mu+\nu)-(c+\phi) P
$$

one obtains

$$
\mathbf{w}=\left[\begin{array}{c}
\beta \\
-(c+\phi)
\end{array}\right], \quad \mathbf{S}=\left[\begin{array}{cc}
0 & 0 \\
1 & 0 \\
0 & -1
\end{array}\right]
$$

To determine matrix $\mathbf{E}$, one needs to evaluate how changing the resident traits changes the equilibrium densities $S$ and $P$. Using implicit differentiation of the equilibrium of system (10), a straightforward but tedious calculation leads to

$$
\mathbf{E}=\left[\begin{array}{ccc}
k e_{11} & e_{12} & e_{13} \\
k \gamma e_{21} & \gamma e_{22} & \gamma e_{23}
\end{array}\right]
$$

where the quantities $e_{i j}$ are given in Appendix B.

To start constructing an example for evolutionary branching in this model, I choose the trait vector $\mathbf{f}=[\alpha, \beta, \phi]^{T}$ such that the resident population has a stable positive equilibrium of its population dynamics and seek a vector $\mathbf{f}^{\prime} \neq \mathbf{0}$ that satisfies $\mathbf{E} \mathbf{f}^{\prime}=\mathbf{0}$. This linear system is underdetermined ( $n=2$ equations with $m=3$ unknowns) and is easily solved to obtain

$$
\begin{aligned}
& f_{2}^{\prime}=f_{1}^{\prime} k \frac{A_{1}}{A_{2}} \\
& f_{3}^{\prime}=f_{1}^{\prime} k \frac{-(c+\phi)}{(b N)^{\prime}-k \alpha-\mu}
\end{aligned}
$$


where

$$
\begin{aligned}
A_{1}= & (b N)^{\prime}((c+\phi) \phi \gamma+\beta \sigma)+ \\
& +(c+\phi)(\beta \delta+\phi \gamma \nu-\beta \gamma N(c+\phi)-\beta K \theta \zeta)-\beta \sigma(k \alpha+\mu) \\
A_{2}= & {\left[(b N)^{\prime}-k \alpha-\mu\right][(c+\phi)(\gamma N(c+\phi)-\delta+K \theta \zeta)+\sigma(k \alpha+\mu+\nu)], }
\end{aligned}
$$

$(b N)^{\prime}$ abbreviates $\frac{d}{d N} b(N) N=b^{\prime}(N) N+b(N)$ and $\sigma=K \theta \zeta^{2} / \rho$. Because $f_{1}^{\prime} \neq 0$ is undetermined, we can set $f_{1}^{\prime}=1$, which is consistent with parameterizing the pathogen strategy with its virulence $(x=\alpha)$.

For a numerical example, I take $b(N)=b_{0}-q N$, fix the parameter values at $b_{0}=3, q=0.3$, $\mu=0.1, \nu=0.2, c=0.2, k=1, \gamma=0.01, \delta=0.05, \theta=0.01, \zeta=0.1, \rho=1, K=15$, and choose the trait values $\alpha=0.1, \beta=1$ and $\phi=1$. This trait vector corresponds to positive equilibrium population densities $(S=2.530, I=2.717, Z=12.337, P=1.775)$, and the equilibrium is locally asymptotically stable (all eigenvalues of the Jacobian have negative real parts). In this example,

$$
\mathbf{f}^{\prime}=\left(\begin{array}{c}
1 \\
2.813 \\
3.447
\end{array}\right)
$$

satisfies $\mathbf{E} \mathbf{f}^{\prime}=\mathbf{0}$. Hence choosing the trade-off functions $\beta(\alpha)$ and $\phi(\alpha)$ according to

$$
\begin{aligned}
& \beta(\alpha)=1+2.813(\alpha-0.1)+\text { h.o.t. } \\
& \phi(\alpha)=1+3.447(\alpha-0.1)+\text { h.o.t. }
\end{aligned}
$$

results in a model that exhibits a singular strategy at the chosen trait vector $(\alpha=0.1, \beta=1$ and $\phi=1$ ) and with $\mathcal{M}=0$ (cf. Proposition 1; see figure 2a).

(a)

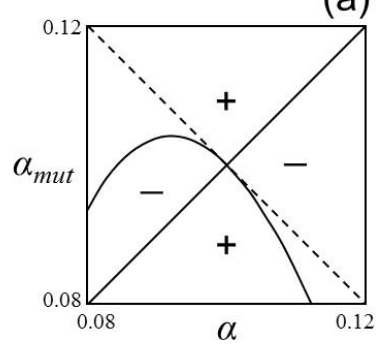

(b)

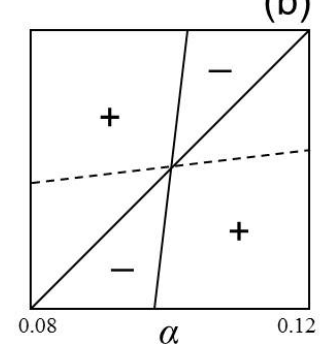

Figure 2: Pairwise invasibility plots of the model in section 4, with parameters as given in the main text and with the trade-off functions in (24) where the higher order terms (h.o.t.) are set to zero. "+" and "-" show the sign of the invasion fitness. (a) $\epsilon=0$, the singularity is degenerate with $\mathcal{M}=0$. The $-45^{\circ}$ secondary diagonal (dashed line) is tangent to the invasion boundary at the singularity, which implies that there is only a cusp (but not a cone) of mutual invasibility attached to the singularity (cf. Geritz et al. 1998). (b) $\epsilon=0.2$, the singularity is an evolutionary branching point. The cone of mutual invasibility is between the invasion boundary and the dashed line. 
The second step of the construction is to change $\mathbf{f}^{\prime}$ into $\mathbf{f}^{\prime}+\Delta \mathbf{f}^{\prime}$ such that the trait vector remains singular but the degeneracy $\mathcal{M}=0$ is unfolded. This requires that $\mathbf{E} \Delta \mathbf{f}^{\prime}$ is orthogonal to $\mathbf{w}$ but not orthogonal to $\mathbf{f}^{\prime} \mathbf{S}$ (cf. Proposition 2). From (13), $\mathbf{E} \Delta \mathbf{f}^{\prime}$ is orthogonal to $\mathbf{w}$ if

$$
\mathbf{E} \Delta \mathbf{f}^{\prime}=\epsilon\left(\begin{array}{c}
c+\phi \\
\beta
\end{array}\right)
$$

This inhomogeneous system is underdetermined with $n=2$ equations and $m=3$ unknowns. However, since we wish to have $\Delta f_{1}^{\prime}=0$ in order to maintain the parameterization $x=\alpha$, we can obtain the remaining unknowns unequivocally as

$$
\Delta \mathbf{f}^{\prime}=\epsilon\left(\begin{array}{c}
0 \\
D_{1} D_{2} /\left(\gamma D_{3}\right) \\
D_{2} /\left(\gamma D_{3}\right)
\end{array}\right)
$$

where $D_{1}, D_{2}$ and $D_{3}$ are shown in Appendix C. In the numerical example, (19) evaluates to

$$
\Delta \mathbf{f}^{\prime}=\epsilon\left(\begin{array}{c}
0 \\
3.831 \\
5.460
\end{array}\right)
$$

According to (9) and using (13) and (18), the change in $\mathcal{M}$ is given by

$$
\Delta \mathcal{M}=\mathbf{f}^{\prime T} \mathbf{S E} \Delta \mathbf{f}^{\prime}=\epsilon \mathbf{f}^{\prime T} \mathbf{S}\left(\begin{array}{c}
c+\phi \\
\beta
\end{array}\right)=\epsilon \mathbf{f}^{\prime T}\left(\begin{array}{c}
0 \\
c+\phi \\
-\beta
\end{array}\right)
$$

which numerically evaluates to a number with sign opposite to that of $\epsilon(\Delta \mathcal{M}=-0.071 \epsilon)$. This means that for sufficiently small positive values of $\epsilon$, the trade-off functions

$$
\begin{aligned}
& \beta(\alpha)=1+(2.813+3.831 \epsilon)(\alpha-0.1)+\text { h.o.t. } \\
& \phi(\alpha)=1+(3.447+5.460 \epsilon)(\alpha-0.1)+\text { h.o.t. }
\end{aligned}
$$

yield a singularity at the same trait vector as above but with $\mathcal{M}<0$, i.e., with a cone of mutual invasibility attached to the singularity (see figure $2 \mathrm{~b}$ ).

The last step of the construction is to find convexities (second derivatives) of the trade-off functions that yield an evolutionary branching point. Given that we already have a singularity with mutual invasibility $(\mathcal{M}<0)$, finding an open set of convexities that yield evolutionary branching is an established procedure (see Proposition 3). Since the invasion fitness in (12) is linear in all three traits, (7) simplifies to $\mathcal{E}=\sum_{i=1}^{m} s_{i} f_{i}^{\prime \prime}$; and since the choice $x=\alpha$ requires $f_{1}^{\prime \prime}=0$, this further simplifies to

$$
\mathcal{E}=S f_{2}^{\prime \prime}-P f_{3}^{\prime \prime}
$$

In order to get evolutionary branching, $f_{2}^{\prime \prime}$ and $f_{3}^{\prime \prime}$ must be chosen such that $0<\mathcal{E}<-\mathcal{M}$ (Table 1), i.e., such that $0<f_{2}^{\prime \prime}-(P / S) f_{3}^{\prime \prime}<-\mathcal{M} / S$ is satisfied. In the numerical example, let us fix $\epsilon=0.2$, which yields $\mathcal{M}=-0.049$ (this value differs from $\Delta \mathcal{M}=-0.071 \epsilon$ because $\Delta \mathcal{M}$ in (9) is only up to linear terms in $\epsilon$, but for the clarity of figure $2 \mathrm{~b}$, I chose $\epsilon$ to be fairly large). With $S=2.530$ and $P=1.775$ (see above), the condition for branching evaluates to 
$0<f_{2}^{\prime \prime}-0.702 f_{3}^{\prime \prime}<0.019$, which is satisfied for example by $f_{2}^{\prime \prime}=-0.01$ and $f_{3}^{\prime \prime}=-0.02$. This means that with $\epsilon=0.2$ in the trade-off functions

$$
\begin{aligned}
& \beta(\alpha)=1+(2.813+3.831 \epsilon)(\alpha-0.1)-\frac{1}{2} \times 0.01(\alpha-0.1)^{2}+\text { h.o.t. } \\
& \phi(\alpha)=1+(3.447+5.460 \epsilon)(\alpha-0.1)-\frac{1}{2} \times 0.02(\alpha-0.1)^{2}+\text { h.o.t. }
\end{aligned}
$$

the model has an evolutionary branching point at $\alpha=0.1$. The higher order terms of these trade-off functions are arbitrary and may be chosen such that the entire function is biologically plausible. Figure 2b shows the resulting branching point with the higher order terms set to zero. Appendix D explains how the pairwise invasibility plot in figure $2 \mathrm{a}$ transforms into figure $2 \mathrm{~b}$ as $\epsilon$ increases from 0 to 0.2 .

In the remainder of this section, I consider three special cases of this model that violate the assumptions of Propositions 1 and 2, respectively.

The case of $k=0$ : not enough traits. If $k$ is zero, then the virulence does not affect the invasion fitness per se, and therefore it is not an observable trait (even though it still mediates a trade-off between $\beta$ and $\phi$ ). Accordingly, the first column of matrix $\mathbf{E}$ in (14) is zero, and to satisfy assumption (A2), $\alpha$ has to be removed from the trait vector $\mathbf{f}$. This means that the condition $m>n$ in Proposition 1 is no longer satisfied. $\mathbf{E}$ becomes a $2 \times 2$ matrix generically of full rank, and therefore the equation $\mathbf{E} \mathbf{f}^{\prime}=\mathbf{0}$ has only the trivial solution $\mathbf{f}^{\prime}=\mathbf{0}$. This is also seen in $(15 \mathrm{a}, \mathrm{b})$, where both elements of $\mathbf{f}^{\prime}$ are zero when $k=0$, i.e., the construction method fails to produce a trade-off structure with $\mathbf{f}^{\prime} \neq \mathbf{0}$ that would yield a singularity with $\mathcal{M}=0$. As we shall see in the next section, with $k=0$ no such trade-off structure exists.

The case of $\gamma=0$ : not enough feedbacks. If $\gamma$ is zero, then even though the predator kills the host, the captured hosts do not contribute to the predator's population growth (the predator feedback loop is not closed). $P$ is then fully determined by the alternative prey, the term $(c+\phi) P$ in the invasion fitness (12) is an externally determined death rate, and $P$ is not a feedback variable. Accordingly, the second row of $\mathbf{E}$ is zero such that $\operatorname{rank}(\mathbf{E})=1$, violating the non-degeneracy condition (i) of Proposition 2. In this case a singularity with $\mathcal{M}=0$ can be constructed, but the degeneracy of $\mathcal{M}=0$ cannot be unfolded. The same is seen in (19), where the denominators vanish when $\gamma=0$, i.e., the unfolding vector does not exist. In fact, the model with $\gamma=0$ is an optimization model (Metz et al. 2008), where evolution minimizes the only remaining feedback variable $S$ or, equivalently, maximizes $\beta /[k \alpha+\mu+\nu+(c+\phi) P]$ (the latter is the basic reproduction number $R_{0}$ of the disease divided with the size of the disease-free host population). In optimization models mutual invasibility and mutual exclusion are impossible, and therefore every singularity is necessarily characterized with $\mathcal{M}=0$.

Violation of $\mathbf{S}^{T} \mathbf{f}^{\prime} \neq \kappa \mathbf{w}$. Given $\mathbf{S}$ and $\mathbf{w}$ in (13) and $\mathbf{f}^{\prime}$ in (15), a straightforward calculation (performed in Mathematica 9.0) shows that $\mathbf{S}^{T} \mathbf{f}^{\prime}=\kappa \mathbf{w}$ for some $\kappa \in \mathbb{R}$ and therefore the nondegeneracy condition (ii) in Proposition 2 is violated if and only if $(b N)^{\prime}=-\nu$. In this case, the vector $\Delta \mathbf{f}^{\prime}$ in (19) does not unfold $\mathcal{M}=0$ because (9) evaluates to zero. 


\subsection{Direct analysis}

Given a concrete and relatively simple model as the one in equations (10), it is possible to calculate $\mathcal{M}$ directly in terms of the slopes of the trade-off functions. This direct analysis gives necessary and sufficient conditions for obtaining a singularity at a given point and with a given sign of $\mathcal{M}$, which can then be used to construct a singularity of a desired type (cf. Table 1). As above, I parameterize the pathogen strains by their virulence and construct the trade-off functions $\alpha \mapsto \beta(\alpha)$ and $\alpha \mapsto \phi(\alpha)$ locally near the given trait values $[\alpha, \beta, \phi]$ to obtain an evolutionary branching point.

From (11), the invasion fitness of a mutant pathogen $\alpha_{m u t}$ in the resident system with strain $\alpha$ is given by

$$
r_{\alpha}\left(\alpha_{m u t}\right)=\beta\left(\alpha_{m u t}\right) S(\alpha)-\left(k \alpha_{m u t}+\mu+\nu\right)-\left(c+\phi\left(\alpha_{m u t}\right)\right) P(\alpha)
$$

where $S(\alpha)$ and $P(\alpha)$ denote respectively the equilibrium densities of susceptibles and of predators in the equilibrium system with the resident strain $\alpha$, obtained from equations (10). In this section, I use the traditional formulation of invasion fitness $r_{\alpha}\left(\alpha_{m u t}\right)$ given as a function of only the resident and mutant strategies (as in Geritz et al. 1998).

The condition for $\alpha$ to be singular is

$$
\left.\frac{\partial r_{\alpha}\left(\alpha_{m u t}\right)}{\partial \alpha_{m u t}}\right|_{\alpha_{m u t}=\alpha}=\beta^{\prime}(\alpha) S(\alpha)-k-\phi^{\prime}(\alpha) P(\alpha)=0
$$

Let us therefore fix

$$
\beta^{\prime}(\alpha)=\frac{k+\phi^{\prime}(\alpha) P(\alpha)}{S(\alpha)}
$$

such that the singularity condition is satisfied. This leaves $\phi^{\prime}(\alpha)$ free to tune the value of $\mathcal{M}$.

The mixed derivative $\mathcal{M}$ is, from its definition,

$$
\mathcal{M}=\left.\frac{\partial^{2} r_{\alpha}\left(\alpha_{m u t}\right)}{\partial \alpha \partial \alpha_{m u t}}\right|_{\alpha_{m u t}=\alpha}=\beta^{\prime}(\alpha) S^{\prime}(\alpha)-\phi^{\prime}(\alpha) P^{\prime}(\alpha)
$$

where $S^{\prime}(\alpha)$ and $P^{\prime}(\alpha)$ denote the derivatives of equilibrium population densities with respect to the resident trait value. These have to be determined from equations (10). First I use the linear equation $\frac{1}{I} \frac{d I}{d t}=0$ to express $S(\alpha)=(k \alpha+\mu+\nu+(c+\phi(\alpha)) P(\alpha)) / \beta(\alpha)$; hence

$$
S^{\prime}(\alpha)=\frac{k+\phi^{\prime}(\alpha) P(\alpha)+(c+\phi(\alpha)) P^{\prime}(\alpha)}{\beta(\alpha)}-\frac{\beta^{\prime}(\alpha) S(\alpha)}{\beta(\alpha)}=\frac{c+\phi(\alpha)}{\beta(\alpha)} P^{\prime}(\alpha)
$$

where in the last step I used the singularity condition (27). A more tedious task is to determine $P^{\prime}(\alpha)$. A lengthy calculation using implicit differentiation and the singularity condition leads to

$$
P^{\prime}(\alpha)=\frac{\gamma I\left[k(c+\phi)-\phi^{\prime} \cdot\left(k \alpha+\mu-(b N)^{\prime}\right)\right]}{\Psi}
$$


where $(b N)^{\prime}=b^{\prime}(N) N+b(N)$ and

$$
\begin{aligned}
\Psi= & \frac{K \theta \zeta^{2}}{\rho}\left[(b N)^{\prime}-(k \alpha+\mu+(c+\phi) P)\right]+ \\
& +\gamma \frac{c+\phi}{\beta}\left[\phi \cdot(b N)^{\prime}-(c+\phi)(\beta I+\mu+c P)-c \nu\right]
\end{aligned}
$$

(here the arguments of functions have been suppressed for brevity, i.e., $\phi=\phi(\alpha), \phi^{\prime}=\phi^{\prime}(\alpha)$, etc.). Substituting (27), (29) and (30) into (28) yields

$$
\begin{aligned}
\mathcal{M}= & \frac{1}{\Psi} \frac{\gamma I(\alpha)}{\beta(\alpha) S(\alpha)}\left[k(c+\phi(\alpha))-\phi^{\prime}(\alpha)(k \alpha+\mu+\nu)\right] \times \\
& \times\left[k(c+\phi(\alpha))-\phi^{\prime}(\alpha)\left(k \alpha+\mu-(b N)^{\prime}\right)\right] .
\end{aligned}
$$

$\mathcal{M}$ is quadratic in $\phi^{\prime}(\alpha)$ with roots $\phi_{1}^{\prime}=\frac{k(c+\phi(\alpha))}{k \alpha+\mu+\nu}$ and $\phi_{2}^{\prime}=\frac{k(c+\phi(\alpha))}{k \alpha+\mu-(b N)^{\prime}}$. If $\Psi$ and $k \alpha+$ $\mu-(b N)^{\prime}$ have the same sign (opposite sign), then $\mathcal{M}$ is a parabola that opens up (down) and therefore it is negative when $\phi^{\prime}(\alpha)$ is inbetween (outside of) $\phi_{1}^{\prime}$ and $\phi_{2}^{\prime}$. (Note that the signs of $\Psi$ and $k \alpha+\mu-(b N)^{\prime}$ can be determined only when the function $b$ is specified.) Hence one can choose $\phi^{\prime}(\alpha)$ such that $\mathcal{M}$ is negative, and choose $\beta^{\prime}(\alpha)$ according to (27) such that $\alpha$ is singular. To complete the construction of an evolutionary branching point, the second derivatives of the trade-off functions must be chosen such that

$$
\mathcal{E}=\left.\frac{\partial^{2} r_{\alpha}\left(\alpha_{m u t}\right)}{\partial \alpha_{m u t}^{2}}\right|_{\alpha_{m u t}=\alpha}=S(\alpha) \beta^{\prime \prime}(\alpha)-P(\alpha) \phi^{\prime \prime}(\alpha)
$$

satisfies $0<\mathcal{E}<-\mathcal{M}$ (see Table 1 ); since both $\beta^{\prime \prime}(\alpha)$ and $\phi^{\prime \prime}(\alpha)$ are free to choose, this can be done with considerable freedom.

In summary, the construction of a branching point consists of the following steps (other types of singularities can be constructed analogously):

1. Choose trait values $\alpha, \beta(\alpha)$ and $\phi(\alpha)$ that correspond to a viable resident system with a stable population dynamical equilibrium; this gives the zero order terms of the Taylorexpansion of the trade-off functions $\beta$ and $\phi$ about $\alpha$, and specifies the densities $S(\alpha)$, $I(\alpha)$ and $P(\alpha)$.

2. Choose the slope $\phi^{\prime}(\alpha)$ of the function $\phi$ at the point $\alpha$ such that $\mathcal{M}$ in (31) is negative and choose $\beta^{\prime}(\alpha)$ according to (27) to satisfy the singularity condition; this gives the first order terms of the trade-off functions.

3. Choose the convexities $\beta^{\prime \prime}(\alpha)$ and $\phi^{\prime \prime}(\alpha)$ such that $\mathcal{E}$ in (32) is positive but less than $-\mathcal{M}$; this gives the second order terms of the trade-off functions.

4. The previous steps ensure that $\alpha$ is an evolutionary branching point. The higher order terms of the trade-off functions can be chosen arbitrarily.

The construction method fails if either $\mathcal{M}$ is identically zero or if the roots $\phi_{1}^{\prime}$ and $\phi_{2}^{\prime}$ coincide (in the latter case, $\mathcal{M}$ is either nonpositive or nonnegative depending on $\Psi$ and $k \alpha+\mu-(b N)^{\prime}$, 
and if nonnegative, then a branching point cannot be obtained). As (31) reveals, $\mathcal{M} \equiv 0$ if $\gamma=0$; and $\phi_{1}^{\prime}=\frac{k(c+\phi(\alpha))}{k \alpha+\mu+\nu}$ and $\phi_{2}^{\prime}=\frac{k(c+\phi(\alpha))}{k \alpha+\mu-(b N)^{\prime}}$ coincide if either $k=0$ or $(b N)^{\prime}=-\nu$ (note that $c+\phi(\alpha)=0$ is irrelevant as it means that the predator attacks neither healthy nor infected hosts). The last three paragraphs of the previous section discussed how these three special cases violate the conditions under which Propositions 1 and 2 hold.

\section{Discussion}

In this paper, I have given sufficient conditions under which one can choose the trade-off functions of a model such that an arbitrarily given point in trait space is an evolutionary singularity of an arbitrary type (for example, a given point is an evolutionary branching point). Under some mild technical conditions, varying the trade-off structure will yield all generic types of evolutionary singularities (listed in Table 1), provided that the model has at least two environmental feedback variables $(n \geq 2)$ and more traits than feedbacks $(m>n)$. Since the traits are determined by an underlying scalar strategy that generates $m-1$ trade-offs between $m$ traits, the second condition can be phrased as having at least as many trade-offs as environmental feedbacks.

The present results significantly extend the scope of critical function analysis. The existing technique (de Mazancourt and Dieckmann 2004; Bowers et al. 2005; Kisdi 2006) considered only a single trade-off linking two traits. With $m=2$, the singularity condition (5) determines $\mathbf{f}^{\prime}$ up to a multiplicative constant, which fixes the sign of $\mathcal{M}$ (cf. (6)); in other words, no flexibility remains to control $\mathcal{M}$. The existing technique was thus restricted to controlling $\mathcal{E}$ via the convexity of the single trade-off, and could construct a given type of singularity only if the sign of $\mathcal{M}$ happened to be appropriate at least at some points in the trait space.

The key to construct an evolutionary singularity of an arbitrary type is to have the mixed derivative $\mathcal{M}$ change its sign. This is more difficult to achieve than controlling $\mathcal{E}$ for two reasons. Firstly, $\mathcal{M}$ does not depend on the second and higher derivatives of the trade-offs, hence only the slopes (first derivatives) of the trade-offs can be used to control $\mathcal{M}$; but the slopes must also satisfy the singularity condition. This explains why at least two trade-offs are (generically) necessary to satisfy the singularity condition and $\mathcal{M}=0$ at a given point. Secondly, $\mathcal{M}$ is quadratic in the slopes of the trade-offs (cf. (6)), and therefore $\mathcal{M}=0$ need not have real roots. The existence of real roots can be ensured when $m>n$ (Proposition 1).

Unfolding the degeneracy of $\mathcal{M}=0$ into an arbitrary sign of $\mathcal{M}$ is straightforward, provided that there are at least two environmental feedbacks $(n \geq 2), m \geq n$, and some non-degeneracy conditions are satisfied (Proposition 2). It is well known that two environmental feedback variables are necessary for the coexistence of two strategies at a population dynamical equilibrium; this is the straightforward extension of the fact that in resource competition, the number of resources limits the number of coexisting competitors (Levin 1970; McGehee and Armstrong 1977; Meszéna et al. 2006). In agreement with this, $\mathcal{M}=0$ can be unfolded only if $n \geq 2$ 
(see Remarks 8-10). Once the sign of $\mathcal{M}$ corresponds to the desired singularity, one can use the existing technique of critical function analysis to find trade-offs yielding the desired singularity (this step is given in Proposition 3 for completeness).

The condition $m>n$ is not necessary for a model to exhibit all generic types of evolutionary singularities. Many simple models have only a single trade-off function connecting two traits. These models do not satisfy $m>n$ with $n \geq 2$, and hence one cannot guarantee that $\mathcal{M}$ changes sign. Indeed, in some of these models $\mathcal{M}$ is always positive (this excludes evolutionary branching; see Kisdi and Meszéna 1995 for an example), in others $\mathcal{M}$ is always negative (in which case critical function analysis can produce examples for evolutionary branching at any point), and yet in others $\mathcal{M}$ changes sign when the position of the singularity is varied, so that all singularities can be achieved at some points in the trait space. Boldin et al. (2009) shows an example with only one trade-off, where seemingly minor details of the model determine whether $\mathcal{M}$ changes sign or not. The direct analysis described in section 4.3 is independent of whether or not the condition $m>n$ is satisfied, and can be used to explore whether $\mathcal{M}$ changes sign in a concrete model (in which case the model can produce all generic types of singularities) or not.

This paper focused on the role of the trade-off functions in generating arbitrary types of evolutionary singularities. Many models have also other functions the shape of which can be chosen with some freedom, so that they could also be used to control $\mathcal{M}$. Kisdi and Boldin (2013) investigated a model for pathogen evolution where the incidence function was used in this way. Models frequently include density-dependent demographic rates, and the function describing density-dependence is also an ideal candidate for this type of analysis. In both of these examples, the argument of the function is an environmental feedback variable, i.e., the invasion fitness can be written in the form $s\left(f_{1}(y), \ldots, f_{m}(y), g\left(E_{1}, \ldots, E_{n}\right), E_{1}, \ldots, E_{n}\right)$ where $g$ is the incidence function, a density-dependent rate of birth or death, etc. Because $\mathcal{M}$ is linear in the first derivatives of $g$, controlling $\mathcal{M}$ via $g$ is actually easier than controlling $\mathcal{M}$ via the trade-offs. To obtain a singularity of arbitrary type, the model needs to have also at least one trade-off, such that the second derivative of this trade-off can be used to control $\mathcal{E}$.

The sufficient conditions described in this paper guarantee the existence of trade-off structures that yield a certain singularity, but it remains to be seen whether these trade-offs are biologically plausible. Obviously, this question can be addressed only in the context of a specific model, where one can define the criteria of biological plausibility. For instance, in the example of section 4, biological considerations suggest that the transmission rate $\beta$ and the predation rate $\phi$ should increase with the virulence of the disease, $\alpha$ (and the trade-off functions in (24) indeed satisfy these criteria when the third and higher order terms are chosen appropriately). With criteria of biological plausibility in hand, one can use the direct analysis shown in section 4.3 to explore the model systematically and see if any plausible trade-off structure yields the desired singularity. To do this, determine locally (i.e., up to second order) all trade-off structures that yield the desired singularity at a given point in trait space; and vary the position of the singularity in trait space as well as the values of scalar parameters to explore all possible trade-offs that yield the desired singularity, checking whether any of them satisfies the plausibility criteria. A negative result proves that the desired singularity cannot occur in systems considered biologically plausible. Note that it would not be possible to prove the same by trying 
to substitute an infinite variety of plausible functions for the trade-offs.

An obvious and important extension of the current work would be to have multi-dimensional trade-offs between the traits, i.e., to allow the traits $\mathbf{f}(\mathbf{x})$ to depend on a vector-valued strategy $\mathbf{x}$ or to consider each trait itself as an element of a vector-valued strategy $(\mathbf{f}(\mathbf{x})=\mathbf{x})$. Obviously, the present model subsumes vector-valued strategies in the sense that with the choice $\mathbf{f}\left(x_{1}, x_{2}, \ldots, x_{k}\right)=\mathbf{f}\left(x_{1}, \cdot, \ldots, \cdot\right)$ (i.e., $\mathbf{f}$ depends on $\mathbf{x}$ only via the scalar $x_{1}$ ), the present model applies directly and the sufficient conditions described in this paper guarantee that the overall evolutionary outcome is as required (for example, that evolutionary branching occurs). The present results are however insufficient to obtain important details of the multivariate evolutionary dynamics. For example, one may wish to have evolutionary branching such that the lineages diverge in both $x_{1}$ and $x_{2}$, or diverge in $x_{1}$ but not in $x_{2}$. This would require the true generalization of the present results to vector-valued strategies, which I must leave to future research because the classification of singularities of vector-valued strategies is still lacking.

\section{Acknowledgements}

I am grateful to two anonymous reviewers, whose comments helped to improve the presentation. This research was financially supported by the Academy of Finland.

\section{References}

[1] Boldin B., Geritz S. A. H. and Kisdi E. 2009. Superinfections and adaptive dynamics of pathogen virulence revisited: a critical function analysis. Evol. Ecol. Res. 11: 153-175.

[2] Bowers R. G., Hoyle A., White A. and Boots M. 2005. The geometric theory of adaptive evolution: Trade-off and invasion plots. J. Theor. Biol. 233, 363-377.

[3] de Mazancourt C. and Dieckmann U. 2004. Trade-off geometries and frequency-dependent selection. Am. Nat. 164: 765-778.

[4] Diekmann O., Gyllenberg M., Huang H., Kirkilionis M., Metz J. A. J. and Thieme H. R. 2001. On the formulation and analysis of general deterministic structured population models. II. Nonlinear theory. J. Math. Biol. 43: 157-189.

[5] Diekmann O., Gyllenberg M. and Metz J. A. J. 2003. Steady state analysis of structured population models. Theor. Pop. Biol. 63: 309-338.

[6] Eshel I. 1983. Evolutionary and continuous stability. J. theor. Biol. 103: 99-111.

[7] Geritz S. A. H. 2005. Resident-invader dynamics and the coexistence of similar strategies. J. Math. Biol. 50: 67-82.

[8] Geritz S. A. H., Gyllenberg M., Jacobs F. and Parvinen K. 2002. Invasion dynamics and attractor inheritance. J. Math. Biol. 44: 548-560. 
[9] Geritz S. A. H. Kisdi E., Meszéna G. and Metz J.A.J. 1998. Evolutionarily singular strategies and the adaptive growth and branching of the evolutionary tree. Evol. Ecol. 12: 35-57.

[10] Geritz S. A. H. Kisdi E. and Yan P. 2007. Evolutionary branching and long-term coexistence of cycling predators: Critical function analysis. Theor. Pop. Biol. 71: 424-435.

[11] Kisdi E. 2006. Trade-off geometries and the adaptive dynamics of two co-evolving species. Evol. Ecol. Res. 8: 959-973.

[12] Kisdi E. and Boldin B. 2013. A construction method to study the role of incidence in the adaptive dynamics of pathogens with direct and environmental transmission. J. Math. Biol. 66: 10211044 .

[13] Kisdi E., Geritz S. A. H. and Boldin B. 2013. Evolution of pathogen virulence under selective predation: A construction method to find eco-evolutionary cycles. J. theor. Biol. 339: 140-150.

[14] Kisdi E. and Meszéna G. 1995. Life histories with lottery competition in a stochastic environment: ESSs which do not prevail. Theor. Pop. Biol. 47: 191-211.

[15] Levin S. 1970. Community equilibria and stability, and an extension of the competitive exclusion principle. Am. Nat. 104: 413-423.

[16] Maynard Smith J. 1982. Evolution and the theory of games. Cambridge University Press, Cambridge.

[17] McGehee R. and Armstrong R. A. 1977. Some mathematical problems concerning the ecological principle of competitive exclusion. J. Diff. Eq. 23: 30-52.

[18] Meszéna G., Gyllenberg M., Pásztor L. and Metz J. A. J. 2006. Competitive exclusion and limiting similarity: A unified theory. Theor. Pop. Biol. 69: 68-87.

[19] Metz J. A. J., Nisbet R. M. and Geritz S. A. H. 1992. How should we define 'fitness' for general ecological scenarios? Trends Ecol. Evol. 7:198-202.

[20] Metz J. A. J., Mylius S. and Diekmann O. 2008. When does evolution optimize? Evol. Ecol. Res. 10: 629-654.

[21] Morozov A. \& A. Best. 2012. Predation on infected host promotes evolutionary branching of virulence and pathogens' biodiversity. J. theor. Biol. 307: 29-36.

[22] Mylius S. D. and Diekmann O. 1995. On evolutionarily stable life histories, optimization and the need to be specific about density dependence. Oikos 74: 218-224.

[23] Priklopil T. 2012. On invasion boundaries and the unprotected coexistence of two strategies. J. Math. Biol. 64: 1137-1156.

[24] Svennungsen T. and Kisdi E. 2009. Evolutionary branching of virulence in a single-infection model. J. theor. Biol. 257: 408-418.

[25] Zu J., Wang K. and Mimura M. 2011. Evolutionary branching and evolutionarily stable coexistence of predator species: Critical function analysis. Math. Biosci. 231: 210-224. 


\section{Appendix A}

In this Appendix, I show that the population dynamical equilibrium of the model in (10) is asymptotically stable if $b^{\prime}(N) \rightarrow-\infty$; the same holds when $b^{\prime}(N)$ is sufficiently large negative. $b^{\prime}(N) \rightarrow-\infty$ implies that the total host density $N$ is constant, and therefore (10) can be replaced with the 3 -dimensional system

$$
\begin{aligned}
\frac{d I}{d t} & =[\beta(N-I)-(k \alpha+\mu+\nu)-(c+\phi) P] I \\
\frac{d Z}{d t} & =[\rho(1-Z / K)-\zeta P] Z \\
\frac{d P}{d t} & =[\gamma c N+\gamma \phi I+\theta \zeta Z-\delta] P
\end{aligned}
$$

The characteristic equation of this system is

$$
-\operatorname{det}\left[\begin{array}{ccc}
-\beta I-\lambda & 0 & -(c+\phi) I \\
0 & -\rho Z / K-\lambda & -\zeta Z \\
\gamma \phi P & \theta \zeta P & -\lambda
\end{array}\right]=\lambda^{3}+a_{1} \lambda^{2}+a_{2} \lambda+a_{3}=0
$$

where

$$
\begin{aligned}
& a_{1}=\beta I+\frac{\rho Z}{K} \\
& a_{2}=\beta I \frac{\rho Z}{K}+\theta \zeta^{2} P Z+\gamma \phi(c+\phi) P I \\
& a_{3}=\beta I \theta \zeta^{2} P Z+\gamma \phi(c+\phi) P I \frac{\rho Z}{K}
\end{aligned}
$$

By the Routh-Hurwitz criteria, all eigenvalues of the Jacobian have negative real parts if $a_{1}>0$, $a_{3}>0$, and $a_{1} a_{2}>a_{3}$. The first two of these conditions obviously hold. For the last, it is easy to verify that

$$
a_{1} a_{2}-a_{3}=\left(\beta I+\frac{\rho Z}{K}\right) \beta I \frac{\rho Z}{K}+\beta I \cdot \gamma \phi(c+\phi) P I+\frac{\rho Z}{K} \theta \zeta^{2} P Z
$$

which is always positive.

\section{Appendix B}

Here I give the elements of matrix $\mathbf{E}$ for the application in section 4 . To find the interior equilibria of the feedback variables, it is convenient to rewrite equations (10) using $N=S+I$ into the form

$$
\begin{aligned}
\frac{d N}{d t} & =b(N) N-(\mu+c P) N-(k \alpha+\phi P) I=0 \\
\frac{1}{I} \frac{d I}{d t} & =\beta(N-I)-(\mu+\nu+k \alpha)-(c+\phi) P=0 \\
\frac{1}{Z} \frac{d Z}{d t} & =\rho(1-Z / K)-\zeta P=0 \\
\frac{1}{P} \frac{d P}{d t} & =\gamma c S+\gamma(c+\phi) I+\theta \zeta Z-\delta=0
\end{aligned}
$$


Equations (35b)-(35d) are linear and thus easily solved for $I, Z$ and $P$ in terms of the still unknown $N$. Substituting the solution into (35a) yields a single nonlinear equation (35a*) for $N$ (not shown), which can be solved only when the density-dependent birth rate function $b$ is specified. Assuming $b(N)=b_{0}-q N$ (i.e., a logistic host), (35a*) is only quadratic, but its solution is an unruly formula.

The elements of $\mathbf{E}$ are the derivatives of the feedback variables $S=N-I$ and $P$ with respect to the traits $\alpha, \beta$ and $\phi$. To obtain these, I differentiate (35a*) implicitly with respect to a trait (e.g. with respect to $\alpha$ to obtain $d N / d \alpha$ ) and obtain the derivatives of the feedbacks (e.g. $d S / d \alpha=d N / d \alpha-d I / d \alpha$ and $d P / d \alpha)$ by differentiating the explicit solution from (35b)-(35d) and substituting the derivative of $N$ from the implicit differentiation. The calculations were done in Mathematica 9.0 and yield $e_{i j}$ in the form

$$
e_{i j}=A_{i j} / B_{i}
$$

with $A_{i j}$ and $B_{i}$ given below (note that $k$ and $\gamma$ have been factored out in (14)).

$$
\begin{aligned}
A_{11}= & -\left(K \rho \theta \zeta^{2}(c+\phi)(\gamma(\alpha c k+c(\mu+\nu)-\phi \nu)+\beta \gamma N(c+\phi)-\beta \delta)+\right. \\
& +\beta K^{2} \theta^{2} \zeta^{4}(\alpha k+\mu)-K \theta \zeta^{2}\left(N b^{\prime}(N)+b(N)\right)\left(\beta K \theta \zeta^{2}+\phi \gamma \rho(c+\phi)\right)+ \\
& \left.+\beta K^{2} \rho \theta^{2} \zeta^{3}(c+\phi)+c \gamma \rho^{2}(c+\phi)^{2}(\gamma N(c+\phi)-\delta)+c \gamma K \rho^{2} \theta \zeta(c+\phi)^{2}\right) \times \\
& \times\left(\beta K \theta \zeta^{2}+\phi \gamma \rho(c+\phi)\right) \\
A_{12}= & -\left(\left(K \theta \zeta^{2}(\alpha k+\mu+\nu)+\rho(c+\phi)(\gamma N(c+\phi)-\delta)+K \rho \theta \zeta(c+\phi)\right) \times\right. \\
& \times\left(-K \rho \theta \zeta^{2}(c+\phi)(-\phi \gamma(\alpha k+\mu+2 \nu)+2 \beta \gamma N(c+\phi)-\beta \delta)-\right. \\
& -\beta K^{2} \theta^{2} \zeta^{4}(\alpha k+\mu)+K \theta \zeta^{2}\left(N b^{\prime}(N)+b(N)\right)\left(\beta K \theta \zeta^{2}+\phi \gamma \rho(c+\phi)\right)- \\
& \left.\left.-\beta K^{2} \rho \theta^{2} \zeta^{3}(c+\phi)-\delta \phi \gamma \rho^{2}(c+\phi)^{2}+\phi \gamma K \rho^{2} \theta \zeta(c+\phi)^{2}\right)\right) \\
& -\left(\rho \left(\left(N b^{\prime}(N)+b(N)\right)\left(\beta K \theta \zeta^{2}+\phi \gamma \rho(c+\phi)\right) \times\right.\right. \\
A_{13}= & \left(K \theta \zeta^{2}(\gamma(c+2 \phi)(\alpha k+\mu+\nu)+\beta(\delta-2 \gamma N(c+\phi))-\right. \\
& \left.-\beta K^{2} \theta^{2} \zeta^{3}+\gamma \rho(c+\phi)^{2}(c \gamma N-\delta)+\gamma K \rho \theta \zeta(c+\phi)^{2}\right)+ \\
& +\beta^{2} K \theta \zeta^{2}(2 \gamma N(c+\phi)-\delta+K \theta \zeta) \times \\
& \times\left(K \theta \zeta^{2}(\alpha k+\mu)+\rho(c+\phi)(\gamma N(c+\phi)-\delta)+K \rho \theta \zeta(c+\phi)\right)+ \\
& +\beta \gamma\left(-K \rho \theta \zeta^{2}(c+\phi)(\alpha k(\gamma N(c+\phi)(c+2 \phi)-\delta(c+3 \phi))+\right. \\
& +c^{2} \gamma \mu N+c(\phi(3 \gamma \mu N+4 \gamma N \nu+K \rho \theta)-\delta \mu)+\phi(-3 \delta(\mu+\nu)+ \\
& +2 \phi \gamma N(\mu+2 \nu)+\phi K \rho \theta)-K^{2} \rho \theta^{2} \zeta^{3}(c+\phi)((c+3 \phi)(\alpha k+\mu)+3 \phi \nu)- \\
& -K^{2} \theta^{2} \zeta^{4}(c+2 \phi)(\alpha k+\mu)(\alpha k+\mu+\nu)- \\
& \left.-\phi K \rho^{2} \theta \zeta(c+\phi)^{2}(\gamma N(c+\phi)-2 \delta)+\delta \phi \rho^{2}(c+\phi)^{2}(\gamma N(c+\phi)-\delta)\right)- \\
& -
\end{aligned}
$$




$$
\begin{aligned}
& -\phi \gamma^{2} \rho(c+\phi)(\rho(c+\phi)(c(\alpha k+\mu)(\gamma N(c+\phi)-\delta)+\delta \phi \nu)+ \\
& \left.\left.\left.+K \rho \theta \zeta(c+\phi)(\alpha c k+c \mu-\phi \nu)+K \theta \zeta^{2}(\alpha k+\mu+\nu)(\alpha c k+c \mu-2 \phi \nu)\right)\right)\right)
\end{aligned}
$$

$$
\begin{aligned}
A_{21}= & -(\rho(\rho(c+\phi)(\phi \gamma(\alpha c k-\phi \mu)+\beta(c+\phi)(c \gamma N-\delta))+ \\
& +\beta K \theta \zeta^{2}(\alpha k(2 c+\phi)-c(\beta N-\mu-\nu)+\phi(\nu-\beta N))+ \\
& \left.\left.+\phi\left(N b^{\prime}(N)+b(N)\right)\left(\beta K \theta \zeta^{2}+\phi \gamma \rho(c+\phi)\right)+\beta K \rho \theta \zeta(c+\phi)^{2}\right)\right)
\end{aligned}
$$

$$
\begin{aligned}
A_{22}= & \rho\left(\alpha c k+\phi N b^{\prime}(N)+\phi b(N)-\phi \mu\right) \times \\
& \times\left(K \theta \zeta^{2}(\alpha k+\mu+\nu)+\rho(c+\phi)(\gamma N(c+\phi)-\delta)+K \rho \theta \zeta(c+\phi)\right)
\end{aligned}
$$

$$
\begin{aligned}
A_{23}= & \rho\left(\rho \left(\alpha^{2} c \phi \gamma k^{2}+\alpha \phi k(\gamma(c(\mu+\nu)-\phi \mu)-\beta \delta)-\mu(\beta \delta(c+2 \phi)-\right.\right. \\
& \left.\left.-\beta \gamma N(c+\phi)^{2}+\phi^{2} \gamma(\mu+\nu)\right)\right)+\left(N b^{\prime}(N)+b(N)\right) \times \\
& \times\left(\rho\left(\phi^{2} \gamma(\alpha k+\mu+\nu)+\beta \delta(c+2 \phi)-\beta \gamma N(c+\phi)^{2}\right)+\right. \\
& \left.+\beta K \theta \zeta^{2}(\beta N-\alpha k-\mu-\nu)-\beta K \rho \theta \zeta(c+2 \phi)\right)+ \\
& \left.+\beta K \rho \theta \zeta(\alpha \phi k+\mu(c+2 \phi))+\beta K \theta \zeta^{2}(\alpha k+\mu)(\alpha k-\beta N+\mu+\nu)\right)
\end{aligned}
$$

$$
\begin{aligned}
B_{1}= & \left(\beta K \theta \zeta^{2}+\phi \gamma \rho(c+\phi)\right) B_{2} \\
B_{2}= & -\beta^{2} K \theta \zeta^{2}\left(K \theta \zeta^{2}(\alpha k+\mu)+\rho(c+\phi)(2 \gamma N(c+\phi)-\delta)+K \rho \theta \zeta(c+\phi)\right)+ \\
& +\beta \gamma \rho(c+\phi)\left(K \theta \zeta^{2}(\alpha k(c+\phi)+2 \phi \nu)-\delta \phi \rho(c+\phi)+\phi K \rho \theta \zeta(c+\phi)\right)+ \\
& +\phi \gamma^{2} \rho^{2}(c+\phi)^{2}(\alpha c k-\phi \mu)+\left(N b^{\prime}(N)+b(N)\right)\left(\beta K \theta \zeta^{2}+\phi \gamma \rho(c+\phi)\right)^{2}
\end{aligned}
$$




\section{Appendix C}

The quantities that appear in (19) are as follows (with $(b N)^{\prime}=b^{\prime}(N) N+b(N)$ and $\left.\sigma=K \theta \zeta^{2} / \rho\right)$ :

$$
\begin{aligned}
D_{1}= & \frac{\beta((c+\phi) \gamma N+K \theta \zeta-\delta)-\gamma \phi(k \alpha+\mu+\nu)}{(c+\phi)^{2} \gamma N+\phi K \theta \zeta+\sigma(k \alpha+\mu+\nu)+c(K \theta \zeta-\delta)-\phi \delta} \\
D_{2}= & \phi \gamma^{2}(c+\phi)^{2}(\phi \mu-k \alpha c)+ \\
& +\beta^{2} \sigma[(c+\phi)(2(c+\phi) \gamma N+K \theta \zeta-\delta)+\sigma(k \alpha+\mu)]- \\
& -\beta \gamma(c+\phi)[\phi(c+\phi)(K \theta \zeta-\delta)+\sigma(k \alpha(c+\phi)+2 \phi \nu)]- \\
& -(b N)^{\prime}(\phi \gamma(c+\phi)+\beta \sigma)^{2} \\
D_{3}= & {\left[(b N)^{\prime}-k \alpha-\mu\right][(c+\phi)(c \gamma N+K \theta \zeta-\delta)-\sigma(\beta N-k \alpha-\mu-\nu)] }
\end{aligned}
$$

\section{Appendix D}

In this Appendix, I explain how the evolutionary singularity in figure 2a bifurcates into the one in figure $2 \mathrm{~b}$ with increasing $\epsilon$ (note in particular that the singularity in figure 2a is an evolutionary repellor, whereas the branching point in figure $2 \mathrm{~b}$ is convergence stable). This bifurcation is not directly relevant to the construction method, because the construction first fixes the value of $\epsilon$ and only then chooses the coefficients of the quadratic terms in (24), the choice depending on $\epsilon$.

The construction method starts with $\mathbf{f}^{\prime}(x)$ such that $x$ is singular with $\mathcal{M}=0$, and then replaces $\mathbf{f}^{\prime}(x)$ with $\mathbf{f}^{\prime}(x)+\Delta \mathbf{f}^{\prime}(x)$ such that $\mathcal{M}$ becomes negative; in the example of (24), the latter is done by replacing $\epsilon=0$ with $\epsilon>0$. By the same replacement, the value of $\mathcal{E}$ changes with

$$
\Delta \mathcal{E}=\sum_{i=1}^{m} \sum_{j=1}^{m} s_{i, j}\left(\Delta f_{i}^{\prime} \cdot f_{j}^{\prime}+f_{i}^{\prime} \cdot \Delta f_{j}^{\prime}\right)
$$

(cf. (7)). In general, $\Delta \mathcal{E}$ may have any sign depending on $s_{i, j}$, the second derivatives of the invasion fitness with respect to traits; this is the reason why $\mathbf{f}^{\prime \prime}(x)$ has to be chosen after fixing $\Delta \mathbf{f}^{\prime}(x)$ (or $\epsilon$ ), such that the final sign of $\mathcal{E}$ is as required by the desired singularity.

In the simple model of section 4, however, the invasion fitness (12) is linear in the traits, such that $s_{i, j}=0$ for all $i, j$ and $\mathcal{E}=\sum_{i=1}^{m} s_{i} f_{i}^{\prime \prime}$ is independent of $\Delta \mathbf{f}^{\prime}(x)$. Increasing $\epsilon$ therefore decreases $\mathcal{M}$ but does not change $\mathcal{E}$. Since the desired singularity is an evolutionary branching point, $\mathbf{f}^{\prime \prime}(x)$ is chosen such that $\mathcal{E}>0$ (cf. Table 1). At $\epsilon=0$, the singularity has $\mathcal{M}=0$ and since $\mathcal{E}+\mathcal{M}>0$, the singularity is not convergence stable (figure 2a). When $\epsilon=\epsilon_{T R}=0.07524$, then $\mathcal{M}=-\mathcal{E}$ and the singularity is not hyperbolic. Because $\Delta \mathbf{f}^{\prime}(x) /\left\|\Delta \mathbf{f}^{\prime}(x)\right\|$ (the direction of $\left.\Delta \mathbf{f}^{\prime}(x)\right)$ is chosen such that the location of the singularity is fixed, convergence stability changes through a transcritical bifurcation (see figure 3). When $\epsilon>\epsilon_{T R}, \mathcal{E}+\mathcal{M}<0$ and the singularity

is convergence stable; and since the value of $\mathcal{E}$ is constant positive for any $\epsilon$, the singularity is a 
branching point (figure 2b).

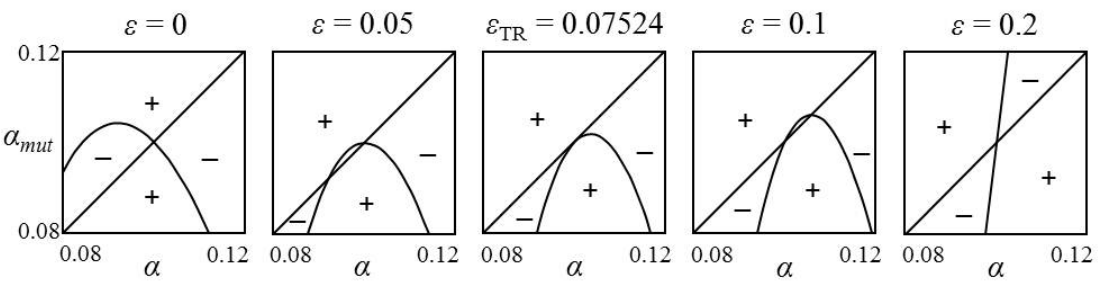

Figure 3: The transcritical bifurcation connecting the two pairwise invasibility plots in figure 2. The outermost panels are identical to figures $2 \mathrm{a}$ and $2 \mathrm{~b}$, respectively. The transcritical bifurcation occurs approximately at $\epsilon=0.07524$. All parameters are as in figure 2 , except $\epsilon$ as shown in the panels. 